\title{
ORTHOGONAL POLYNOMIALS, MEASURES AND RECURRENCES ON THE UNIT CIRCLE
}

\author{
PAUL NEVAI
}

\begin{abstract}
New characterizations are given for orthogonal polynomials on the unit circle and the associated measures in terms of the reflection coefficients in the recurrence equation satisfied by the polynomials.
\end{abstract}

1. Introduction. Let $d \mu$ be a finite positive Borel measure on the interval $[0,2 \pi)$ with an infinite set as its support, and let $\left\{\varphi_{n}\right\}(n=0,1, \ldots)$ be the unique system of orthonormal polynomials on the unit circle associated with this measure. That is the polynomials

$$
\varphi_{n}(z)=\varphi_{n}(d \mu, z)=\kappa_{n} z^{n}+\cdots \quad\left(\kappa_{n}=\kappa_{n}(d \mu)>0\right)
$$

are such that

$$
(2 \pi)^{-1} \int_{0}^{2 \pi} \varphi_{m}(z) \overline{\varphi_{n}(z)} d \mu(\theta)=\delta_{m n} \quad(z=\exp (i \theta) ; m \in \mathbf{N}, n \in \mathbf{N}) .
$$

The monic orthogonal polynomials $\Phi_{n}$ are defined by

$$
\Phi_{n}(z)=\Phi_{n}(d \mu, z)=\kappa_{n}^{-1} \varphi_{n}(z) .
$$

The reverse polynomial $\Pi^{*}$ of a polynomial $\Pi$ of degree $n$ is given by

$$
\Pi^{*}(z)=z^{n} \bar{\Pi}\left(z^{-1}\right)
$$

In the theory of orthogonal polynomials a central role is played by the two dual pairs of recurrence formulas

$$
\kappa_{n-1} \varphi_{n}(z)=z \kappa_{n} \varphi_{n-1}(z)+\varphi_{n}(0) \varphi_{n-1}^{*}(z)
$$

and

$$
\kappa_{n-1} \varphi_{n}^{*}(z)=\kappa_{n} \varphi_{n-1}^{*}(z)+z \overline{\varphi_{n}(0)} \varphi_{n-1}(z),
$$

or

$$
\Phi_{n}(z)=z \Phi_{n-1}(z)+\Phi_{n}(0) \Phi_{n-1}^{*}(z)
$$

Received by the editors December 5, 1985 and, in revised form, April 10, 1986.

1980 Mathematics Subject Classification (1985 Revision). Primary 42C05.

Key words and phrases. Orthogonal polynomials, recurrence formulas, difference equations, Szegö Theory.

This material is based upon work supported by the National Science Foundation under Grant No. DMS 84-19525, by the United States Information Agency under Senior Research Fulbright Grant No. 85-41612, by the Hungarian Ministry of Education, and by the Catholic University of Louvain in Louvain-La-Neuve, Belgium. 
and

$$
\Phi_{n}^{*}(z)=\Phi_{n-1}^{*}(z)+z \overline{\Phi_{n}(0)} \Phi_{n-1}(z),
$$

$n=1,2, \ldots$ (cf. [Sz, formula (11.4.7), p. 293]). Since all zeros of the orthogonal polynomials lie inside the unit circle (cf. [Sz, Theorem 11.4.1, p. 292]) the reflection coefficients $\Phi_{n}(0)$ satisfy

$$
\left|\Phi_{n}(0)\right|<1, \quad n=1,2, \ldots .
$$

The connection between the reflection coefficients $\Phi_{n}(0)$ and the leading coefficients $\kappa_{n}$ of the orthogonal polynomials is given by

$$
\kappa_{n}=\kappa_{0} \prod_{k=1}^{n}\left[1-\left|\Phi_{k}(0)\right|^{2}\right]^{-1 / 2}
$$

(cf. [Ger1, formula (8.6), p. 156]). These recurrence formulas fully characterize the orthogonal polynomials and the associated measures. This fact is expressed by the analogue of J. Favard's theorem for the unit circle according to which any system of polynomials $\left\{\Phi_{n}\right\}$ satisfying (1.3) and (1.5) is orthogonal with respect to a unique (up to a positive constant factor) measure $d \mu$ (cf. [Ger1, Theorem 8.1, p. 156]).

Due to this intimate connection between the orthogonal polynomials, the corresponding measure and the associated recurrence formula, and due to their wide applicability in diverse areas such as approximation theory, digital signal processing, electrical engineering, nuclear physics, prediction theory, statistical physics, statistics, scattering theory, and solid state physics, there has been a great amount of research dedicated to characterizing the measures and the corresponding orthogonal polynomials in terms of the reflection coefficients $\Phi_{n}(0)$. The first result of this nature was obtained by S. N. Bernstein and G. Szegö whose papers implicitly contain the interesting observation that $\Phi_{n}(0)=0$ for all but a finite number of $n$ if and only if the measure is absolutely continuous and its absolutely continuous component equals the reciprocal of a positive trigonometric polynomial (cf. [ $\mathbf{S z}$, $\S 2.6$, pp. 31-33 and Theorem 11.2, p. 289; Fr, Theorem V.4.5, p. 224]). The full characterization of measures $d \mu$ for which $\sum\left|\Phi_{n}(0)\right|<\infty$ was started by Ya. L. Geronimus (cf. [Ger 1-3]) and it was completed by G. Baxter [Ba]. Subsequently, A. Bultheel, K. M. Case, Ph. Delsarte, Y. Genin, J. S. Geronimo, M. Kac, G. López, A. Máté, E. M. Nikishin, V. Totik, the author and others obtained a variety of results regarding the properties of measures that can be determined from various properties of the reflection coefficients $\Phi_{n}(0)$ (cf. [Bu, Ca2, DeGe1, 2, Ge, GeCa1, Ger4, Ló, MáNe2, MáNeTo1, 2, 4, 5, 6, Ne1, 6, 7, NeTo and Ni]).

Historically, such investigations were preceded by results of direct nature which characterize the reflection coefficients $\Phi_{n}(0)$ in terms of the measure $d \mu$. The two most important results were obtained by G. Szegö, Ya. L. Geronimus, and E. A. Rachmanov who respectively proved that

$$
\log \mu^{\prime} \in L_{1}[0,2 \pi] \Leftrightarrow \sum_{k=1}^{\infty}\left|\Phi_{k}(0)\right|^{2}<\infty
$$


(cf. [Sz, Lemma, p. 300] for " $\Rightarrow$ " and [Ger1, Theorem 8.2, p. 160 or Ak, Corollary

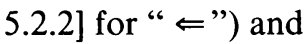

$$
\mu^{\prime}>0 \text { a.e. } \Rightarrow \lim _{n \rightarrow \infty} \Phi_{n}(0)=0
$$

(cf. [Ra1, 1 , p. 207; Ra2, Theorem, p. 106; MáNe2; MáNeTo2, Theorem 1, p. 64]).

In view of (1.7) and (1.8) one would like to obtain results describing the measure $d \mu$ provided that (in addition to some other conditions) either $\lim \Phi_{n}(0)=0$ or $\Sigma\left|\Phi_{k}(0)\right|^{2}<\infty$ hold. For $\lim \Phi_{n}(0)=0$ this was initiated by A. Máté, V. Totik, and the author in [MáNeTo1,4,5,6 and Ne6] where we laid the foundations of a theory of orthogonal polynomials that goes beyond the Szegö Theory (cf. [Ne7]). The case $\sum\left|\Phi_{k}(0)\right|^{2}<\infty$ was recently examined by E. M. Nikishin [Ni] who found a couple of interesting properties of the orthogonal polynomials and the corresponding measures in terms of the reflection coefficients $\Phi_{n}(0)$. He proved

Proposition 1.1 [Ni, TheOrem 1, P. 397]. Let $\Sigma\left|\Phi_{k}(0)\right|^{2}<\infty$. Then there exists $a$ constant $C>0$ such that

$$
|\log | \Phi_{n}\left(e^{i \theta}\right)|| \leqslant C\left[1+\sum_{k=1}^{n+1}\left|\Phi_{k}(0) \sum_{j=k}^{n+1} \Phi_{j}(0) e^{i j \theta}\right|\right]
$$

holds for $n=1,2, \ldots$ and $\theta \in[0,2 \pi]$.

Proposition 1.2 [Ni, Corollary 1, P. 397 and Remark, P. 399]. Assume that the reflection coefficients $\Phi_{n}(0)$ are real, $\Sigma \Phi_{n}^{2}(0)<\infty$, and $\Phi_{n}(0)$ tends to zero monotonically as $n \rightarrow \infty$. Then $d \mu$ is absolutely continuous in the open interval $(0,2 \pi)$ with a possible mass point at $\theta=0$.

The purpose of this paper is to continue the investigation by E. M. Nikishin and to improve these two results. Among others, we will show that if the conditions of Proposition 1.2 are satisfied, then the absolutely continuous component $\mu^{\prime}$ of $d \mu$ is a positive and continuous function in the open interval $(0,2 \pi)$, and if, in addition, $\Phi_{n}(0) \downarrow 0$, then $d \mu$ is absolutely continuous in its entire domain $[0,2 \pi)$.

Since it is easier to work with analytic functions in the unit disk than with such functions in the complex domain cut along an interval, results on complex orthogonal polynomials usually precede analogous results on orthogonal polynomials associated with measures on the real line. Nevertheless, there are exceptions to this rule, and such an example is given by the above quoted results of E. M. Nikishin whose real line analogues were found by K. M. Case, J. M. Dombrowski, G. H. Fricke, J. S. Geronimo, A. Máté, V. Totik, and the author (see [Ca1, GeCa2 and Ne1, 2, 4, 5] for analogues of Proposition 1.1 and [Do1-4, DoFr, DoNe, MáNe3, MáNeTo3, 7 and Ne2, 4] for Proposition 1.2).

Inequality (1.9) is fairly delicate in the sense that the right-hand side is strongly sensitive on the location of the point $\theta$. On the other hand, the unit circle is rotation invariant, and thus the natural (or possibly better described as unsophisticated) approach yields uniform estimates for both the orthogonal polynomials and the related quantities. As opposed to the unit circle, real intervals and orthogonal 
polynomials on them require highly sophisticated methods even if one wants to prove simple analogues of results that are valid for complex orthogonal polynomials. This need for sophistication explains why the theorems analogous to Propositions 1 and 2 and the tools to prove them developed before E. M. Nikishin's paper [Ni].

E. M. Nikishin must have been unaware of the considerable amount of research concerning the link between orthogonal polynomials defined by recurrence formulas and the corresponding measures (cf. [AsIs, Ch, Ga, Ger1, 4, Ne1, 5,7] and the references therein). Having read his paper [Ni] and having understood his techniques, we immediately know that not only can Propositions 1 and 2 be improved upon, but a variety of results analogous to real orthogonal polynomials can also be proved by systematically exploiting the simple (Abel's transformation, i.e., summation by parts) but still ingenious (one has to apply it at the right time) idea that lies behind the proof of Proposition 1.1.

The Szegö Theory is concerned with the behavior of orthogonal polynomials when $\log \mu^{\prime}$ is integrable, and the principal tool is the Szegö function $D(d \mu)$ which is defined by

$$
D(d \mu, z)=\exp \left\{(4 \pi)^{-1} \int_{0}^{2 \pi} \log \mu^{\prime}(\theta)\left(e^{i \theta}+z\right)\left(e^{i \theta}-z\right)^{-1} d \theta\right\}, \quad|z|<1 .
$$

The nontangential boundary value of $D(d \mu)$ on the unit circle exists almost everywhere, and we will denote it also by $D(d \mu)=D\left(d \mu, e^{i \theta}\right)$. We have $\left|D\left(d \mu, e^{i \theta}\right)\right|^{2}=\mu^{\prime}(\theta)$ almost everywhere. One way of stating the fundamental result of the Szegö Theory is that

$$
\lim _{n \rightarrow \infty} \int_{0}^{2 \pi}\left|e^{-i n \theta} \varphi_{n}\left(e^{i \theta}\right)-\bar{D}^{-1}\left(d \mu, e^{-i \theta}\right)\right|^{2} \mu^{\prime}(\theta) d \theta=0
$$

(cf. [Sz, Chapters X and XI; Fr, formula V.4.1, p. 219; Ne7, §4.11]).

2. The results. In what follows the functions $s_{m}$ are defined by

$$
s_{m}(z)=\sum_{k=1}^{m} \overline{\Phi_{k}(0)} z^{k}, \quad s_{-1}=0 .
$$

The meat of the matter is the following inequality that can be proved by following and properly modifying the steps used by E. M. Nikishin to prove Proposition 1.1.

THEOREM 2.1. Let the (monic) orthogonal polynomial system $\left\{\Phi_{n}\right\}$ satisfy the recurrence formula (1.3), i.e.

$$
\Phi_{n}(z)=z \Phi_{n-1}(z)+\Phi_{n}(0) \Phi_{n-1}^{*}(z) .
$$

Let $n=1,2, \ldots$, and let $f$ be an arbitrary function defined on the unit circle. Then the inequality

$$
\begin{aligned}
|\log | \Phi_{n}\left(e^{i \theta}\right)|| \leqslant & 4 \sum_{k=0}^{n-1}\left|\Phi_{k}(0)\left[s_{k}\left(e^{i \theta}\right)-f\left(e^{i \theta}\right)\right]\right| \\
& +\left|s_{n}\left(e^{i \theta}\right)-f\left(e^{i \theta}\right)\right|+\sum_{k=1}^{n}\left|\Phi_{k}(0)\right|^{2}\left(1-\left|\Phi_{k}(0)\right|\right)^{-1}
\end{aligned}
$$

holds for $\theta \in[0,2 \pi]$. 
Setting $f=s_{n}$ here and assuming the convergence of the series $\sum\left|\Phi_{n}(0)\right|^{2}$ we obtain Proposition 1.1. An even more interesting case is when $f$ is the (possibly formal) power series whose partial sums are given by (2.1).

Proposition 1.2 can be improved and generalized as follows.

THEOREM 2.2. Let the (monic) orthogonal polynomial system $\left\{\Phi_{n}\right\}$ satisfy the recurrence formula (2.2). Assume the reflection coefficients $\Phi_{n}(0)$ satisfy

$$
\lim _{n \rightarrow \infty} \Phi_{n}(0)=0
$$

and

$$
\sum_{k=1}^{\infty}\left|\Phi_{k}(0) \sum_{m=k}^{\infty}\right| \Phi_{m+1}(0)-\Phi_{m}(0)||<\infty .
$$

Then the measure $d \mu$ can be written as

$$
d \mu(\theta)=\mu^{\prime}(\theta) d \theta+\delta(\theta)
$$

where $\delta$ is a nonnegative mass point at 0 and $\mu^{\prime}$ is a positive continuous function in the open interval $(0,2 \pi)$. In addition, $\log \mu^{\prime}$ is integrable and the Szegö function $D(d \mu)$ is also continuous in the closed unit disk centered at the origin with the exception of perhaps the point 1. Finally, for the orthonormal polynomials $\varphi_{n}$ the asymptotic formula

$$
\lim _{n \rightarrow \infty} e^{-i n \theta} \varphi_{n}\left(e^{i \theta}\right)=\bar{D}^{-1}\left(d \mu, e^{-i \theta}\right)
$$

holds uniformly on every closed set of $(0,2 \pi)$.

Using a simple rotation of the unit circle, analogous results can be stated and proved when the argument of an arbitrary point $z$ with $|z|=1$ is the only potential candidate for a mass point in (2.6).

In terms of the reverse orthogonal polynomial $\varphi_{n}^{*}(2.7)$ is equivalent to $\lim \varphi_{n}^{*}(z)$ $=D^{-1}(d \mu, z)$ uniformly in $z$ if $z$ belongs to any arc of the unit circle not containing the point 1 . By the Szegö Theory this also holds uniformly on every compact set in the open unit disk (cf. [Sz, Theorem 12.1.1, p. 297]) Certainly, if the conditions of Theorem 2.2 are satisfied, then $\lim \varphi_{n}^{*}(z)=D^{-1}(d \mu, z)$ remains true uniformly on every compact set belonging to the closed unit disk with the point 1 removed. We would be thrilled to see a proof of this.

In the framework of the Szegö Theory, (2.7) is proved provided that the measure satisfies certain assumptions. At the present time, the most general such condition is given by G. Freud [Fr, Theorem V.4.4, p. 223] who proved (2.7) whenever $d \mu$ is absolutely continuous in a neighborhood, say $\Delta$, of $\theta, 0<c<\mu^{\prime}(t)<C<\infty$ for $t \in \Delta$ and

$$
\int_{0}^{2 \pi}\left[\mu^{\prime}(\theta)-\mu^{\prime}(t)\right]^{2}[\theta-t]^{-2} d t<\infty
$$

The following theorem shows that (2.7) is true also if the function $F$, defined by the Fourier series

$$
F(\theta) \sim \sum_{k=0}^{\infty} \overline{\Phi_{k}(0)} e^{i k \theta},
$$

satisfies a similar condition. 
THEOREM 2.3. Let the (monic) orthogonal polynomial system $\left\{\Phi_{n}\right\}$ satisfy the recurrence formula (2.2). Assume the function $F$ in (2.8) is square integrable in $[0,2 \pi]$ and let

$$
\int_{0}^{2 \pi}|F(\theta)-F(t)|^{2}[\theta-t]^{-2} d t<\infty
$$

hold for $\theta \in \mathscr{M} \subset(0,2 \pi)$. Then for every $\theta \in \mathscr{M}$, the limit

$$
\lim _{n \rightarrow \infty} e^{-i n \theta} \varphi_{n}\left(e^{i \theta}\right)=G(\theta)
$$

exists and $G(\theta)=\bar{D}^{-1}\left(d \mu, e^{-i \theta}\right)$ for almost every $\theta \in \mathscr{M}$. Moreover, $\mu^{\prime}>0$ almost everywhere on the set $\mathscr{M}$.

In [Ne3, Theorem, p. 474] it was proved that one can obtain asymptotics not only for the orthogonal polynomials but also for all their derivatives under Freud's conditions. We wonder whether this can be accomplished if the assumptions of Theorem 2.3 hold. It would be interesting to find out the right conditions in terms of the reflection coefficients $\Phi_{n}(0)$ for the existence of $\lim e^{-i n \theta} \varphi_{n}^{(k)}\left(e^{i \theta}\right)(n \rightarrow \infty)$.

Drs. Philip Delsarte and Yves Genin [DeGe2] were kind to point out that the ultraspherical reflection coefficients, that is the reflection coefficients of the ultraspherical measure $d \mu_{0}(\theta)=|\sin (\theta / 2)|^{2 \gamma} d \theta\left(\gamma>-\frac{1}{2}\right)$ on the unit circle, are given by $\Phi_{n}(0)=\gamma /(n+\gamma)$. Hence it is natural to ask whether such a behavior of the reflection coefficients necessarily leads to orthogonal polynomials and measures which closely resemble the ultraspherical polynomials and weight function. For real orthogonal polynomials this problem was solved in [Ne2,4]. For orthogonal polynomials on the unit circle the situation is more complicated since neither $\Phi_{n}(0)=$ $O(1 / n)$ nor $\Phi_{n}(0) \uparrow(\downarrow) 0$ is sufficient for the weight function to exhibit a behavior similar to $|\sin (\theta / 2)|^{2 \gamma}$. For instance, it is easy to see that the reflection coefficients of another type of ultraspherical measure $d \mu_{1}(\theta)$ which is defined by $d \mu_{1}(\theta)=$ $|\sin \theta|^{2 \gamma} d \theta$ satisfy $\Phi_{n}\left(d \mu_{1}, 0\right)=O(1 / n)$, and still $\mu_{1}^{\prime}$ has a singularity at $\theta=\pi$ as well, contrary to the behavior of $\mu_{0}^{\prime}$. This can be verified by using the relationship between the real ultraspherical polynomials in $[-1,1]$ and the polynomials $\Phi_{n}\left(d \mu_{1}\right)$ (cf. [Sz, formula (11.5.2), p. 294]). On the other hand, $\Phi_{n}(0) \uparrow(\downarrow) 0$ is clearly not even sufficient for the integrability of $\log \mu^{\prime}$ (cf. (1.7)), and thus a priori one cannot expect to be able to compare the weight with the ultraspherical one. The following theorem shows that if the reflection coefficients satisfy a weak version of both a monotonicity and a $O(1 / n)$ condition, then the comparison between the appropriate measures and orthogonal polynomials is no longer impossible.

THEOREM 2.4. Let the (monic) orthogonal polynomial system $\left\{\Phi_{n}\right\}$ satisfy the recurrence formula (2.2). Assume that there cxist two positive constants $C$ and $\varepsilon$ such that the reflection coefficients $\Phi_{n}(0)$ satisfy (2.4),

$$
\sum_{k=1}^{n}\left|\Phi_{k}(0)\right|<C \log (n+1) \text {, }
$$


$n=1,2, \ldots$, and

$$
A=\sum_{k=1}^{\infty}(k+1)^{\varepsilon}\left|\Phi_{k}(0) \sum_{m=k}^{\infty}\right| \Phi_{m+1}(0)-\Phi_{m}(0)||<\infty .
$$

Then there are two positive numbers $K \leqslant 1+(3 A)^{[C / \varepsilon]^{*}}$ and $\gamma \leqslant[C / \varepsilon]^{*}$ such that

$$
\left|\varphi_{n}\left(e^{i \theta}\right)\right| \leqslant K|\sin (\theta / 2)|^{-\gamma}
$$

and

$$
\mu^{\prime}(\theta) \geqslant K^{-2}|\sin (\theta / 2)|^{2 \gamma}
$$

uniformly for $n=1,2, \ldots$ and $\theta \in(0,2 \pi)$.

The symbol $[C / \varepsilon]^{*}$ above denotes the ceiling function of $C / \varepsilon$, that is the smallest integer which is at least $C / \varepsilon$. In the above described example of the ultraspherical measure $d \mu_{0}$ the constants $C$ and $\varepsilon$ in (2.11) and (2.12) can be taken as $C=|\gamma|+\delta$ and $\varepsilon=1-\delta$, where $0<\delta<1$ is arbitrarily small. Thus (2.14) yields $\mu_{0}^{\prime}(\theta) \geqslant$ $K^{-2}|\theta|^{[2|\gamma|+\bar{\delta}]^{*}}$, where $\bar{\delta}$ can be chosen as small as one wishes. This shows the nearly optimal sharpness of Theorem 2.4. As was pointed out after Theorem 2.2, the role of $\theta=0$ can be played by any other point $\theta$ in the interval $[0,2 \pi)$ provided that condition (2.12) is properly recasted as well.

Dr. Alphonse Magnus is thanked at this point, who read a preliminary draft of this paper and computed the reflection coefficients of the Jacobi measure

$$
d \mu_{1}(\theta)=|\sin (\theta / 2)|^{2 \gamma_{1}}|\cos (\theta / 2)|^{2 \gamma_{2}} d \theta \quad\left(\gamma_{1}, \gamma_{2}>-\frac{1}{2}\right)
$$

on the unit circle. Magnus' formula [Ma] is $\Phi_{n}(0)=\left(\gamma_{1}+(-1)^{n} \gamma_{2}\right) /\left(n+\gamma_{1}+\gamma_{2}\right)$. In connection with this it is pointed out that the proof of Theorem 2.4 given in $\$ 4$ can be applied to prove a corresponding generalization of Theorem 2.4 which would permit singularities to occur at a finite number of equidistant $(\bmod 2 \pi)$ points.

Although the proof of the following result is almost tirvial, it is still important in that it provides an easy way to rule out the possibility of mass points.

THEOREM 2.5. If the reflection coefficients $\Phi_{n}(0)$ in the recurrence formula (2.2) are real, and they are nonnegative for all but finitely many values of $n$, then the corresponding measure $d \mu$ has no mass point at 0 .

3. The tools. The primary tools are the recurrence formulas (1.1)-(1.4) and their various forms. We will formulate two useful identities as

LEMMA 3.1. The orthogonal polynomials satisfy

$$
\Phi_{n}^{*}(z)=\prod_{k=1}^{n}\left(1+z^{k} \overline{\Phi_{k}(0)} \overline{\Phi_{k-1}^{*}(z)} / \Phi_{k-1}^{*}(z)\right), \quad|z|=1,
$$

and

$$
\begin{aligned}
\kappa_{n} \varphi_{n}^{*}(z)= & \sum_{k=0}^{n}\left(f(z)-s_{k-1}(z)\right) \varphi_{k}(0) \overline{\varphi_{k}(z)} \\
& +\left(s_{n}(z)-f(z)\right) \kappa_{n} \overline{\varphi_{n}^{*}(z)}, \quad|z|=1,
\end{aligned}
$$


for $n=1,2, \ldots$, where $s_{m}$ is given by $(2.1)$; that is

$$
s_{m}(z)=\sum_{k=0}^{m} \overline{\Phi_{k}(0)} z^{k}, \quad s_{-1}=0
$$

and $f$ is an arbitrary function on the unit circle.

Proof. If we rewrite (1.4) as

$$
\Phi_{n}^{*}(z)=\Phi_{n-1}^{*}(z)\left(1+z \overline{\Phi_{n}(0)} \Phi_{n-1}(z) / \Phi_{n-1}^{*}(z)\right),
$$

then applying (3.4) repeatedly we obtain

$$
\Phi_{n}^{*}(z)=\prod_{k=1}^{n}\left(1+z \overline{\Phi_{k}(\hat{v})} \Phi_{k-1}(z) / \Phi_{k-1}^{*}(z)\right)
$$

(cf. [Ger1, formula (8.19), p. 160]). If $|z|=1$, then $z^{-k} \Phi_{k}(z)=(\bar{z})^{k} \Phi_{k}\left(\overline{z^{-1}}\right)$ $=\overline{\Phi_{k}^{*}(z)}$, and hence (3.1) follows from (3.5). To prove (3.2) we use another recurrence analogous to (1.2) which is

$$
\kappa_{n} \varphi_{n}^{*}(z)=\kappa_{n-1} \varphi_{n-1}^{*}(z)+\overline{\varphi_{n}(0)} \varphi_{n}(z)
$$

(cf. [Sz, formula (11.4.6), p. 293]). This can also be applied repeatedly to yield

$$
\kappa_{n} \varphi_{n}^{*}(z)=\sum_{k=0}^{n} \overline{\varphi_{k}(0)} \varphi_{k}(z)
$$

Again, if $|z|=1$, then $z^{-k} \varphi_{k}(z)=(\bar{z})^{k} \varphi_{k}\left(\overline{z^{-1}}\right)=\overline{\varphi_{k}^{*}(z)}$. Thus (3.7) can be rewritten as

$$
\begin{aligned}
\kappa_{n} \varphi_{n}^{*}(z) & =\sum_{k=0}^{n} \overline{\varphi_{k}(0)} z^{k} \overline{\varphi_{k}^{*}(z)} \\
& =\sum_{k=0}^{n} \overline{\Phi_{k}(0)} z^{k}\left[\kappa_{k} \overline{\varphi_{k}^{*}(z)}\right], \quad|z|=1 .
\end{aligned}
$$

Here on the right-hand side we can apply (3.7) one more time to the expression in the brackets. We obtain

$$
\kappa_{n} \varphi_{n}^{*}(z)=\sum_{k=0}^{n} \overline{\Phi_{k}(0)} z^{k} \sum_{m=0}^{k} \varphi_{m}(0) \overline{\varphi_{m}(z)}, \quad|z|=1 .
$$

Let $s_{m}$ be given by (3.3), and let $f$ be an arbitrary function on the unit circle. Then we can rewrite (3.9) as

$$
\kappa_{n} \varphi_{n}^{*}(z)=\sum_{k=0}^{n}\left[\left(s_{k}(z)-f(z)\right)-\left(s_{k-1}(z)-f(z)\right)\right] \sum_{m=0}^{k} \varphi_{m}(0) \overline{\varphi_{m}(z)}
$$

$|z|=1$. Now summation by parts directly leads to (3.2).

LEMMA 3.2. The orthogonal polynomials satisfy

$$
\left|\overline{\Phi_{k}^{*}(z)} / \Phi_{k}^{*}(z)-\overline{\Phi_{k-1}^{*}(z)} / \Phi_{k-1}^{*}(z)\right|<4\left|\Phi_{k}(0)\right|, \quad|z|=1,
$$

for $k=1,2, \ldots$ 
Proof. Applying (3.1) and its complex conjugate with $n=k$ and $n=k-1$ we obtain

$$
\overline{\Phi_{k}^{*}(z)} / \Phi_{k}^{*}(z)=\overline{\Phi_{k-1}^{*}(z)} / \Phi_{k-1}^{*}(z) \frac{1+(\bar{z})^{k} \Phi_{k}(0) \Phi_{k-1}^{*}(z) / \overline{\Phi_{k-1}^{*}(z)}}{1+z^{k} \overline{\Phi_{k}(0)} \overline{\Phi_{k-1}^{*}(z)} / \Phi_{k-1}^{*}(z)} .
$$

Hence

$$
\begin{aligned}
\left|\overline{\Phi_{k}^{*}(z)} / \Phi_{k}^{*}(z)-\overline{\Phi_{k-1}^{*}(z)} / \Phi_{k-1}^{*}(z)\right| & =\left|\left(1+\varepsilon_{k}\right) /\left(1+\bar{\varepsilon}_{k}\right)-1\right| \\
& =\left|1+\varepsilon_{k}\right|\left|\varepsilon_{k}-\bar{\varepsilon}_{k}\right|\left(1+\left|\varepsilon_{k}\right|^{2}\right)^{-1}
\end{aligned}
$$

where $\varepsilon_{k}=(\bar{z})^{k} \Phi_{k}(0) \Phi_{k-1}^{*}(z) / \overline{\Phi_{k-1}^{*}(z)}$. Since $\left|\varepsilon_{k}\right|=\left|\Phi_{k}(0)\right|<1$, the lemma follows immediately.

4. The proofs. The first proof below is essentially a purified version and slight modification of E. M. Nikishin's proof of Proposition 1.1.

Proof of THEOREM 2.1. In what follows we denote the principal value of the logarithmic function by "Log”. Since for $|z|=1$ we have

$$
\left|z^{k} \overline{\Phi_{k}(0)} \overline{\Phi_{k-1}^{*}(z)} / \Phi_{k-1}^{*}(z)\right|=\left|\Phi_{k}(0)\right|<1,
$$

we can take the logarithm of both sides of (3.1) to obtain

$$
\log \left|\Phi_{n}^{*}(z)\right|=\operatorname{Re}\left\{\sum_{k=1}^{n} \log \left(1+z^{k} \overline{\Phi_{k}(0)} \overline{\Phi_{k-1}^{*}(z)} / \Phi_{k-1}^{*}(z)\right)\right\},
$$

$|z|=1$. We proceed with estimating the right-hand side of (4.2) by applying the inequality $|\log (1+\zeta)-\zeta| \leqslant|\zeta|^{2}(1-\mid \zeta)^{-1},|\zeta|<1$, which follows immediately from the Taylor series expansion of the function $\log (1+\zeta)$. Taking (4.1) into consideration as well, we obtain

$$
\begin{aligned}
|\log | \Phi_{n}^{*}(z)|| \leqslant & \left|\sum_{k=1}^{n} z^{k} \overline{\Phi_{k}(0)} \overline{\Phi_{k-1}^{*}(z)} / \Phi_{k-1}^{*}(z)\right| \\
& +\sum_{k=1}^{n}\left|\Phi_{k}(0)\right|^{2}\left(1-\left|\Phi_{k}(0)\right|\right)^{-1}, \quad|z|=1 .
\end{aligned}
$$

The next step is estimating the first sum on the right-hand side of (4.3) by performing summation by parts on it. Let $s_{k}$ be defined by formula (2.1) (cf. (3.3)) and let $f$ be an arbitrary function on the unit circle. Then

$$
\begin{aligned}
\sum_{k=1}^{n} z^{k} & \overline{\Phi_{k}(0)} \overline{\Phi_{k-1}^{*}(z)} / \Phi_{k-1}^{*}(z) \\
= & \sum_{k=1}^{n}\left[\left(s_{k}(z)-f(z)\right)-\left(s_{k-1}(z)-f(z)\right)\right] \overline{\Phi_{k-1}^{*}(z)} / \Phi_{k-1}^{*}(z) \\
= & \sum_{k=1}^{n-1}\left[s_{k}(z)-f(z)\right]\left[\overline{\Phi_{k-1}^{*}(z)} / \Phi_{k-1}^{*}(z)-\overline{\Phi_{k}^{*}(z)} / \Phi_{k}^{*}(z)\right] \\
& -(1-f(z))+\left(s_{n}(z)-f(z)\right) \overline{\Phi_{n-1}^{*}(z)} / \Phi_{n-1}^{*}(z), \quad|z|=1 .
\end{aligned}
$$


Thus by Lemma 3.2 (cf. (3.11))

$$
\begin{aligned}
& \left.\mid \sum_{k=1}^{n} z^{k} \overline{\Phi_{k}(0)} \overline{\Phi_{k-1}^{*}(z)} / \Phi_{k-1}^{*}(z)\right) \mid \\
& \quad \leqslant 4 \sum_{k=1}^{n-1}\left|s_{k}(z)-f(z)\right|\left|\Phi_{k}(0)\right|+|1-f(z)|+\left|s_{n}(z)-f(z)\right|
\end{aligned}
$$

for $|z|=1$. Since, on the unit circle, polynomials and their reverses have the same magnitude, inequality (2.3) follows from (4.3) and (4.4), and so does the theorem.

Proof of Theorem 2.2. By (2.4) and (2.5), $\sum\left|\Phi_{k}(0)\right|^{2}<\infty$ holds. Thus by a theorem of Ya. L. Geronimus $\log \mu^{\prime} \in L^{1}[0,2 \pi]$ (cf. " $\Leftarrow "$ in (1.7)). Hence on the basis of the Szegö Theory we have

$$
\lim _{n \rightarrow \infty} \int_{0}^{2 \pi}\left|\varphi_{n}^{*}\left(e^{i \theta}\right)-D^{-1}\left(d \mu, e^{i \theta}\right)\right|^{2} \mu^{\prime}(\theta) d \theta=0
$$

(cf. (2.10)). Let $f$ be defined by

$$
f(z)=\sum_{k=0}^{\infty} \overline{\Phi_{k}(0)} z^{k}
$$

Then by (2.4) and (2.5) $f$ is analytic for $|z|<1$ and $f$ is continuous on the unit circle with the possible exception of the point 1 . Let $s_{m}$ be the partial sum of the Taylor series in (4.6) (cf. (2.1)). Then summation by parts leads to

$$
\begin{aligned}
f(z) & -s_{k}(z)=\sum_{m=k+1}^{\infty} \overline{\Phi_{m}(0)} z^{m} \\
& =(z-1)^{-1}\left\{\sum_{m=k+1}^{\infty}\left[\overline{\Phi_{m}(0)}-\overline{\Phi_{m+1}(0)}\right]\left[z^{m+1}-1\right]-\overline{\Phi_{k+1}(0)}\left[z^{k+1}-1\right]\right\}
\end{aligned}
$$

so that

$$
\left|f\left(e^{i \theta}\right)-s_{k}\left(e^{i \theta}\right)\right| \leqslant 2(\sin (\theta / 2))^{-1} \sum_{m=k+1}^{\infty}\left|\Phi_{m}(0)-\Phi_{m+1}(0)\right|
$$

holds for $k=0,1, \ldots$ Now applying (1.5), (2.5), and Theorem 2.1 (cf. (2.3) with $f$ given by (4.6)) we obtain that the monic orthogonal polynomials $\Phi_{n}$ and their reverses $\Phi_{n}^{*}$ satisfy

$$
\left|\Phi_{n}(z)\right|^{ \pm 1}=O(1) \text { and }\left|\Phi_{n}^{*}(z)\right|^{ \pm 1}=O(1)
$$

uniformly for $n \in \mathbf{N}$ in every closed arc of the unit circle not containing the point 1 . It follows from (4.9), $\Sigma\left|\Phi_{k}(0)\right|^{2}<\infty$, and (1.6) that we also have

$$
\left|\varphi_{n}(z)\right|^{ \pm 1}=O(1) \text { and }\left|\varphi_{n}^{*}(z)\right|^{ \pm 1}=O(1)
$$

uniformly for $n \in \mathbf{N}$ in every closed arc of the unit circle not containing the point 1 . Once we have established the uniform boundedness of the orthogonal polynomials, we can apply Lemma 3.1 (cf. (3.2) with $f$ given by (4.6)), inequality (4.8), formula (1.6), and conditions (2.4) and (2.5) to conclude that $\lim \varphi_{n}^{*}\left(e^{i \theta}\right)$ exists uniformly in every closed subset of $(0,2 \pi)$. By (4.5) this limit must be $D^{-1}\left(d \mu, e^{i \theta}\right)$, and 
consequently we have proved (2.7). From (4.10) and (2.7) the continuity of $D^{ \pm 1}\left(d \mu, e^{i \theta}\right)$ follows for every $\theta \neq 0$. Since $D(d \mu)$ is the Poisson integral of its nontangential boundary values $\left(D(d \mu) \in H^{2}\right.$ in the unit disk), $D(d \mu)$ is also continuous for all $z \neq 1$ such that $|z| \leqslant 1$. Now the continuity and positivity of $\mu^{\prime}$ follow from the formula $\left|D\left(d \mu, e^{i \theta}\right)\right|^{2}=\mu^{\prime}(\boldsymbol{\theta})$ (cf. [Sz, formula (10.2.8), p. 276]). What remains to be proved is the absolute continuity of the measure $d \mu$ away from $\theta=0$. In view of (4.10) this follows immediately from the fact that the first $n$ moments of $d \mu(\theta)$ and $\left|\varphi_{n}\left(e^{i \theta}\right)\right|^{-2} d \theta$ coincide (cf. [Fr, Theorem V.2.2, p. 198]). Indeed, by this theorem

$$
\lim _{n \rightarrow \infty} \int_{0}^{2 \pi} G(\theta)\left|\varphi_{n}\left(e^{i \theta}\right)\right|^{-2} d \theta=\int_{0}^{2 \pi} G(\theta) d \mu(\theta)
$$

for every continuous function $G$. Thus by (4.10) if $G$ vanishes in a fixed neighborhood, say $\Delta$, of 0 , then

$$
\left|\int_{0}^{2 \pi} G(\theta) d \mu(\theta)\right| \leqslant C\left|\int_{0}^{2 \pi} G(\theta) d \theta\right|,
$$

where the constant $C$ depends only on $\Delta$. Thus $d \mu$ is absolutely continuous outside $\Delta$. Since $\Delta(\ni 0)$ is arbitrary, $(2.6)$ follows, and so does the theorem.

Proof of TheOrem 2.3. We start with proving that if condition (2.9) is satisfied (see (2.1), (2.8), and (4.6) for the definitions of $F, s_{k}$, and $f$, respectively) for $0<\theta<2 \pi$, then

$$
\sum_{k=0}^{\infty}\left|f\left(e^{i \theta}\right)-s_{k}\left(e^{i \theta}\right)\right|^{2}<\infty
$$

holds. Since $f \in H^{2}$ in the unit disk and the integral in (2.9) converges, we have $\left[f(z)-f\left(e^{i \theta}\right)\right]\left[z-e^{i \theta}\right]^{-1} \in H^{2}$ as well (cf. [Fr, Lemma V.3.6, p. 218]), and then

$$
\int_{0}^{2 \pi}[F(\theta)-F(t)]\left[e^{i \theta}-e^{i t}\right]^{-1} e^{i t} d t=0
$$

(N.B. $\left.f\left(e^{i}\right)=F(\cdot)\right)$. But then straightforward computation yields

$$
f\left(e^{i \theta}\right)-s_{k}\left(e^{i \theta}\right)=e^{i(k+1) \theta}(2 \pi)^{-1} \int_{0}^{2 \pi}[F(\theta)-F(t)]\left[e^{i \theta}-e^{i t}\right]^{-1} e^{-i k t} d t
$$

from which, by Bessel's inequality, we obtain

$$
\sum_{k=0}^{\infty}\left|f\left(e^{i \theta}\right)-s_{k}\left(e^{i \theta}\right)\right|^{2}<(2 \pi)^{-1} \int_{0}^{2 \pi}|F(\theta)-F(t)|^{2}\left|e^{i \theta}-e^{i t}\right|^{-2} d t
$$

Now (4.11) follows from (2.9) and (4.12). Applying (4.11) to Theorem 2.1 (cf. inequality (2.3)) and using Schwarz' inequality in the first sum on the right-hand side of (2.3) we obtain

$$
\left|\Phi_{n}\left(e^{i \theta}\right)\right|^{ \pm 1}=O(1) \text { and }\left|\Phi_{n}^{*}\left(e^{i \theta}\right)\right|^{ \pm 1}=O(1)
$$

for $n \in \mathbf{N}$, and then by (1.6)

$$
\left|\varphi_{n}\left(e^{i \theta}\right)\right|^{ \pm 1}=O(1) \text { and }\left|\varphi_{n}^{*}\left(e^{i \theta}\right)\right|^{ \pm 1}=O(1)
$$


$n \in \mathbf{N}$, holds as well. Once we establish (4.13), we can apply (1.6), (4.11), and Schwarz' inequality again to formula (3.2) in Lemma 3.1, and this proves the existence of the limit in (2.10). If (2.9) holds for all $\theta \in \mathscr{M}$, then so does (2.10) on $\mathscr{M}$. In view of (1.7), the square integrability of $F$ implies the integrability of $\log \mu^{\prime}$ so that Szegö's theorem (1.11) yields the connection between the function $G$ in (2.10) and the Szegö function $D(d \mu)$ (cf. (1.10)) which is given by $G(\theta)=\bar{D}^{-1}\left(d \mu, e^{-i \theta}\right)$ for almost every $\theta \in \mathscr{M}$. Finally, by (4.13) $G$ does not vanish on $\mathscr{M}$, so that $\mu^{\prime}$ is positive almost everywhere there.

Proof of Theorem 2.4. In what follows let $0<\theta<2 \pi$. First we will prove inequality (2.13). Note that (2.4) and (2.12) imply the convergence of the series $\sum\left|\Phi_{k}(0)\right|^{2}$, and thus by (1.6) and (1.7) $\log \mu^{\prime}$ is integrable and

$$
0<\lim _{n \rightarrow \infty} \kappa_{n}<\infty \text {. }
$$

Applying (3.1) we obtain the inequality

$$
\left|\Phi_{n}\left(e^{i \theta}\right)\right| \leqslant \exp \left(\sum_{k=1}^{n}\left|\Phi_{k}(0)\right|\right),
$$

$n=1,2, \ldots$, so that by (2.11) and (4.14) we obtain the initial estimate

$$
\left|\varphi_{n}\left(e^{i \theta}\right)\right| \leqslant(n+1)^{C},
$$

$n=1,2, \ldots$. Next we apply Lemma 3.1 (cf. (3.2)-(3.3)) with $f=s_{n}$. We obtain

$$
\left|\varphi_{n}\left(e^{i \theta}\right)\right| \leqslant \sum_{k=0}^{n}\left|s_{n}\left(e^{i \theta}\right)-s_{k-1}\left(e^{i \theta}\right)\right|\left|\Phi_{k}(0) \| \varphi_{k}\left(e^{i \theta}\right)\right|,
$$

$n=1,2, \ldots$ (Here we also used the fact that $\kappa_{n}$ is an increasing sequence, cf. (1.6), and thus $\left|\varphi_{k}(0)\right| \leqslant \kappa_{n}\left|\Phi_{k}(0)\right|$ for $k \leqslant n$.) Writing the expression $s_{n}\left(e^{i \theta}\right)-s_{k-1}\left(e^{i \theta}\right)$ as a sum (cf. (3.3)), using $\lim \Phi_{n}(0)=0$, and performing summation by parts similarly to (4.7) we obtain

$$
\left|s_{n}\left(e^{i \theta}\right)-s_{k-1}\left(e^{i \theta}\right)\right| \leqslant 3(\sin (\theta / 2))^{-1} \sum_{m=k}^{\infty}\left|\Phi_{m}(0)-\Phi_{m+1}(0)\right|
$$

for $k \leqslant n$. Inequalities (4.16) and (4.17) immediately yield

$$
\left|\varphi_{n}\left(e^{i \theta}\right)\right| \leqslant 3(\sin (\theta / 2))^{-1} \sum_{k=0}^{n}\left|\Phi_{k}(0) \sum_{m=k}^{\infty}\right| \Phi_{m}(0)-\Phi_{m+1}(0)||\left|\varphi_{k}\left(e^{i \theta}\right)\right|,
$$

$n=1,2, \ldots$ Here $\left|\varphi_{k}\left(e^{i \theta}\right)\right|$ on the right-hand side can be estimated by (4.15), and we obtain

$$
\left|\varphi_{n}\left(e^{i \theta}\right)\right| \leqslant 3(\sin (\theta / 2))^{-1} \sum_{k=0}^{n}(k+1)^{c}\left|\Phi_{k}(0) \sum_{m=k}^{\infty}\right| \Phi_{m}(0)-\Phi_{m+1}(0)||,
$$

$n=1,2, \ldots$ Thus by (2.12) we can improve (4.15) to

$$
\left|\varphi_{n}\left(e^{i \theta}\right)\right| \leqslant 3 A(n+1)^{C-\varepsilon}(\sin (\theta / 2))^{-1},
$$

$n=1,2, \ldots$ Now, in view of (2.12), we can repeatedly apply (4.20) and the resulting new improved estimates of $\left|\varphi_{n}\left(e^{i \theta}\right)\right|$ to the right-hand side of (4.18). After, say, $L$ new steps we obtain

$$
\left|\varphi_{n}\left(e^{i \theta}\right)\right| \leqslant(3 A)^{L+1}(n+1)^{C-\varepsilon(L+1)}(\sin (\theta / 2))^{-(L+1)},
$$


$n=1,2, \ldots$ Thus we can make $L=[C / \varepsilon-1]$ such steps (here $[\cdot]$ denotes the integer part of the number ".") when we arrive at either

$$
\left|\varphi_{n}\left(e^{i \theta}\right)\right| \leqslant(3 A)^{C / \varepsilon}(\sin (\theta / 2))^{-(C / \varepsilon)}
$$

$n=1,2, \ldots$, if $C / \varepsilon-1$ is an integer, or

$$
\left|\varphi_{n}\left(e^{i \theta}\right)\right| \leqslant(3 A)^{[C / \varepsilon]}(n+1)^{C-\varepsilon[C / \varepsilon]}(\sin (\theta / 2))^{-[C / \varepsilon]},
$$

$n=1,2, \ldots$, if $C / \varepsilon-1<[C / \varepsilon-1]^{*}(=[C / \varepsilon])$. In the latter case we apply (4.23) one more time to the right-hand side of (4.18), and in view of (2.12) we obtain

$$
\left|\varphi_{n}\left(e^{i \theta}\right)\right| \leqslant(3 A)^{[C / \varepsilon]^{*}}(\sin (\theta / 2))^{-[C / \varepsilon]^{*}},
$$

$n=1,2, \ldots$ Since either (4.22) or (4.24) holds, we have proved (2.13). As mentioned before, $\log \mu^{\prime}$ is integrable. Hence we have

$$
\liminf _{n \rightarrow \infty} n^{-1} \sum_{k=0}^{n-1}\left|\varphi_{k}\left(e^{i \theta}\right)\right|^{2}=\left[\mu^{\prime}(\theta)\right]^{-1}
$$

for almost every $\theta \in[0,2 \pi]$ (cf. [MáNe1, Theorem 1, p. 147]). Thus (2.14) follows from (2.13) via (4.25).

Proof of TheOREM 2.5. If the reflection coefficients are real, then by any one of the recurrence formulas (1.1)-(1.4) the orthogonal polynomials have real coefficients so that values at 1 are real. But then by formula (3.1) in Lemma 3.1 we have

$$
\Phi_{n}^{*}(1)=\prod_{k=1}^{n}\left(1+\Phi_{k}(0)\right)
$$

and since the reflection coefficients $\Phi_{k}(0)$ are nonnegative for $k$ sufficiently large, $\lim \Phi_{n}^{*}(1)=0(n \rightarrow \infty)$ cannot be true. Hence by formula (1.6) the orthonormal polynomials also satisfy $\lim \inf \left|\varphi_{n}(1)\right|>0(n \rightarrow \infty)$ so that $\sum\left|\varphi_{n}(1)\right|^{2}=\infty$. However, it is well known from the theory of moments that the divergence of the latter series excludes the possibility for the point 0 to be a mass point for the measure $d \mu$. More accurately, we have $\sum\left|\varphi_{n}(1)\right|^{2}=2 \pi[\mu(\{0\})]^{-1}$ (cf. [Ak, Theorem 2.5 .2 and ShTa, Corollary 2.6, pp. 45-46]).

\section{REFERENCES}

[Ak] N. I. Akhiezer, The classical moment problem, Oliver and Boyd, Edinburgh, 1965.

[AsIs] R. Askey and M. Ismail, Recurrence relations, continued fractions and orthogonal polynomials, Mem. Amer. Math. Soc. No. 300 (1984).

[Ba] G. Baxter, A convergence equivalence related to polynomials orthogonal on the unit circle, Trans. Amer. Math. Soc. 99 (1961), 471-487.

[Bu] A. Bultheel, Convergence of Schur parameters and transmission zeros of a meromorphic spectrum, Proceed. MTNS, 1985.

[Ca1] K. M. Case, Orthogonal polynomials revisited, Theory and Applications of Special Functions (R. A. Askey, ed.), Academic Press, New York, 1975, pp. 289-304.

[Ca2] __ Scattering theory and polynomials orthogonal on the unit circle, J. Math. Phys. 20 (1979), 299-310.

[Ch] T. S. Chihara, An introduction to orthogonal polynomials, Gordon and Breach, New York, 1978.

[DeGe1] Ph. Delsarte and Y. Genin, The split Levinson algorithm, IEEE Trans. Acoust. Speech Signal Process. (to appear).

[DeGe2] , Application of the split Levinson algorithm: the ultraspherical polynomials, manuscript. 
[Do1] J. M. Dombrowski, Spectral properties of phase operators, J. Math. Phys. 15 (1974), 576-577.

[Do2] , Spectral properties of real parts of weighted shift operators, Indiana Univ. Math. J. 29 (1980), 249-259.

[Do3] , Tridiagonal matrix representations of cyclic self-adjoint operators. I, Pacific J. Math. 114 (1984), 325-334.

[Do4] _ Tridiagonal matrix representations of cyclic self-adjoint operators. II, Pacific J. Math. 120 (1985), 47-53.

[DoFr] J. M. Dombrowski and G. H. Fricke, The absolute continuity of phase operators, Trans. Amer Math. Soc. 213 (1975), 363-372.

[DoNe] J. M. Dombrowski and P. Nevai, Orthogonal polynomials, measures and recurrence relations, SIAM J. Math. Anal. 17 (1986), 752-759.

[Fr] G. Freud, Orthogonal polynomials, Pergamon Press, New York, 1971.

[Ga] W. Gautschi, Computational aspects of three-term recurrence relations, SIAM Rev. 9 (1967), 24-82.

[Ge] J. S. Geronimo, Matrix orthogonal polynomials on the unit circle, J. Math. Phys. 22 (1981), 1359-1365.

[GeCa1] J. S. Geronimo and K. M. Case, Scattering theory and polynomials orthogonal on the unit circle, J. Math. Phys. 20 (1979), 299-320.

[GeCa2] , Scattering theory and polynomials orthogonal on the real line, Trans. Amer. Math. Soc. 258 (1980), 467-494.

[Ger1] Ya. L. Geronimus, Orthogonal polynomials, Consultants Bureau, New York, 1961.

[Ger2] (1962), 1-78.

[Ger3] _ On asymptotic properties of polynomials which are orthogonal on the unit circle, and on certain properties of positive harmonic functions, Amer. Math. Soc. Transl. 3 (1962), 79-106.

[Ger4] _ Orthogonal polynomials, Amer. Math. Soc. Transl. 108 (1977), 37-130.

[Ló] G. López Lagomasino, On the asymptotics of the ratio of orthogonal polynomials and convergence of multi-point Padé approximations, Mat. Sb. 128 (170) (1985), 216-229.

[Ma] Al. Magnus, Reflection coefficients of the circular Jacobi polynomials, private communication.

[MáNe1] A. Máté and P. Nevai, Bernstein's inequality in $L_{p}$ for $0<p<1$ and $(C, 1)$ bounds for orthogonal polynomials, Ann. of Math. (2) 111 (1980), 145-154.

[MáNe2] _ Remarks on E. A. Rahmanov's paper "On the asymptotics of the ratio of orthogonal polynomials", J. Approximation Theory 36 (1982), 64-72.

[MáNe3] __ Orthogonal polynomials and absolutely continuous measures, Approximation Theory, IV (C. K. Chui et al., eds.), Academic Press, New York, 1983, pp. 611-617.

[MáNeTo1] A. Máté, P. Nevai, and V. Totik, What is beyond Szegö's theory of orthogonal polynomials, Rational Approximation and Interpolation (P. R. Graves-Morris et al., eds.), Lecture Notes in Math., vol. 1105, Springer-Verlag, New York, 1984, pp. 502-510.

[MáNeTo2] __ Asymptotics for the ratio of leading coefficients of orthonormal polynomials on the unit circle, Constructive Approximation 1 (1985), 63-69.

[MáNeTo3] A. Máté, P. Nevai and V. Totik, Asymptotics for orthogonal polynomials defined by a recurrence relation, Constructive Approximation 1 (1985), 231-248.

[MáNeTo4] Strong and weak convergence of orthogonal polynomials, Amer. J. Math. (to appear).

[MáNeT05] ___ Extensions of Szegö's theory of orthogonal polynomials. II, Constructive Approximation 3 (1987).

[MáNeTo6] , Extensions of Szegö's theory of orthogonal polynomials. III, Constr. Approx. 3 (1987)

[MáNeTo7] , Twisted difference operators and perturbed Chebyshev polynomials, manuscript.

[Ne1] P. Nevai, Orthogonal polynomials, Mem. Amer. Math. Soc., No. 213 (1979).

$[\mathrm{Ne2}]$ , On orthogonal polynomials, J. Approximation Theory 25 (1979), 34-37.

[Ne3] _ An asymptotic formula for the derivatives of orthogonal polynomials, SIAM J. Math. Anal. 10 (1979), 472-477.

[Ne4] _ Orthogonal polynomials defined by a recurrence relation, Trans. Amer. Math. Soc. 250 (1979), 369-384.

[Ne5] _ Two of my favorite ways of obtaining asymptotics for orthogonal polynomials, Functional Analysis and Approximation (P. L. Butzer et al., eds.), ISNM 65, Birkhäuser Verlag, Basel, 1984, pp. 417-436. 
[Ne6] _ Extensions of Szegö's theory of orthogonal polynomials, Orthogonal Polynomials and Their Applications (C. Brezinski et al., eds.), Lecture Notes in Math., vol. 1171, Springer-Verlag, Berlin, 1985, pp. 230-238.

[Ne7] , "Géza Freud, orthogonal polynomials and Christoffel functions, J. Approximation Theory 48 (1986).

[NeTo] P. Nevai and V. Totik, Orthogonal polynomials and their zeros, manuscript.

[Ni] E. M. Nikishin, On an estimate for orthogonal polynomials, Acta Sci. Math. (Szeged) 48 (1985), 395-399. (Russian)

[Ra1] E. A. Rahmanov, On the asymptotics of the ratio of orthogonal poynomials, Math. USSR Sb. 32 (1977), 199-213.

[Ra2] , On the asymptotics of the ratio of orthogonal polynomials. II, Math. USSR Sb. 46 (1983), $105-117$.

[ShTa] J. A. Shohat and J. D. Tamarkin, The problem of moments, Math. Surveys, No. 1, Amer. Math. Soc., Providence, R. I., 1970.

[Sz] G. Szegö, Orthogonal polynomials, Amer. Math. Soc. Colloq. Publ., vol. 23, Amer. Math. Soc., Providence, R. I., 1939, 4th ed., 1975.

Department of Mathematics, Ohio State University, Columbus, Ohio 43210 